\title{
A Review of Low Power Wide Area Technology in Licensed and Unlicensed Spectrum for IoT Use Cases
}

\author{
Noor Laili Ismail ${ }^{1}$, Murizah Kassim², Mahamod Ismail ${ }^{3}$, Roslina Mohamad ${ }^{4}$ \\ ${ }^{1,2,4}$ Faculty of Electrical Engineering, Universiti Teknologi MARA (UiTM), 40450 Shah Alam, Selangor, Malaysia \\ ${ }^{3}$ Department of Electrical, Electronics \& System Engineering, Faculty of Engineering and Built Environment, \\ Universiti Kebangsaan Malaysia (UKM) 43600 Bangi, Selangor, Malaysia
}

\begin{tabular}{l} 
Article Info \\
\hline Article history: \\
Received Feb 10, 2018 \\
Revised Apr 16, 2018 \\
Accepted Apr 30, 2018 \\
\hline
\end{tabular}

\section{Keywords:}

IoT

LoRa

LPWA

LTE-M

NB-IoT

Sigfox

\begin{abstract}
There are many platforms in licensed and license free spectrum that support LPWA (low power wide area) technology in the current markets. However, lack of standardization of the different platforms can be a challenge for an interoperable IoT environment. Therefore understanding the features of each technology platform is essential to be able to differentiate how the technology can be matched to a specific IoT application profile. This paper provides an analysis of LPWA underlying technology in licensed and unlicensed spectrum by means of literature review and comparative assessment of Sigfox, LoRa, NB-IoT and LTE-M. We review their technical aspect and discussed the pros and cons in terms of their technical and other deployment features. General IoT application requirements is also presented and linked to the deployment factors to give an insight of how different applications profiles is associated to the right technology platform, thus provide a simple guideline on how to match a specific application profile with the best fit connectivity features.
\end{abstract}

Copyright $@ 2018$ Institute of Advanced Engineering and Science. All rights reserved.

\section{Corresponding Author:}

Noor Laili Ismail

Faculty of Electrical Engineering, Universiti Teknologi MARA (UiTM), 40450 Shah Alam, Selangor, Malaysia.

E-mail: lailiis@yahoo.co.uk

\section{INTRODUCTION}

IoT has become the notion of the future internet in which smart sensors, devices and everyday objects are connected and communicated with each other without human intervention, and extends the capability into smart object networking, a scenario that has help to set the groundwork for today's IoT. The goal of IoT is to bring smart objects or devices together anytime, anywhere in a universal network, creating an intelligent system [2]. IoT has shifted computing paradigm from human oriented to autonomous smart devices, commonly in monitoring, remote diagnostics \& control, leading to cost savings. As IoT proliferates, it will have an impact on the network traffic distribution, characteristics and performance [3], [4]. IoT will demand a wide range of new technologies and skills, a new cloud service, a new network and hardware platform, a new type of high volume data processing and so on. IoT innovation is geared to offer a wide spectrum of new services, generate new market segments and revenues. This will result in high increasing demand of network capacity by years [5], and even some of its various use cases is expected to introduce different requirements on connectivity economically and technically. The advent of LPWA technology at this point of time is seems to back the instances, for few primary reasons such as cost, coverage and power consumption [6].

Supporting vasts connected devices that will potentially be mounted in remote areas require a very low-end device cost and easy to install, not only because the number of devices could be huge, but also to minimize the risk of theft. The need for a high capacity gateway is also important to limit the number of 
gateways, thus reduced the infrastructure cost. High power efficiency devices are as well critical to prolong the network lifespan and avoid high battery maintenance cost. In IoT landscape especially in rural area, monitoring might be placed in an area without wired infrastructure with no or very poor access to any cellular base station. Therefore, the technology must be able to provide a good coverage service to enable connectivity of the devices. The ability to scale efficiently and support diverse IoT requirements from different use cases would also become important for massive IoT deployment in the long run. In physic law, low power and wide coverage can be achieved by trading off the bit rate. This marks the unique characteristic of LPWA technology as compared to traditional network connectivity in IoT landscape such as Bluetooth, Zig-Bee, Wi-Fi and cellular [7].

\section{RESEARCH METHOD}

This paper provides an analysis of some LPWA underlying technology namely Sigfox, LoRa, NBIoT and LTE-M, its business model and ecosystem in licensed and unlicensed spectrum. Section 2 starts with the technology evolution and the technical features of each. Section 3 addresses each technology features and shortly discussed the pros and cons of each in terms of deployment factors. The nature of various IoT applications is also listed to relate to the deployment factors and give an insight of different application requirements against the right technology platform. This can serve as a guideline on how to match the best-fit technology for specific application needs. Section 4 concludes the discussion with a general observation of the market trend and current situation of LPWA landscape for IoT.

The technology that covers long range communication is somehow new. The drive began with Sigfox, followed by LoRa and others in unlicensed band spectrum. With the recent announcement of 3GPP standard release 12 and 13, LPWA technology is set to enter a new phase as cellular carriers are also offering their IoT connectivity options via LTE-M and NB-IoT in 2017. Starting from here, LTE-M will be rolling out its initial IoT connectivity in the US, while NB-IoT will initiate in Europe. This marks a significant technology jump into IoT landscape on LPWAN. In LPWAN protocol, NB-IoT and LTE-M are using licensed spectrum while Sigfox and LoRaWAN are using license free spectrum. Currently, there are many license exempt spectrums exist in the market for LPWAN platform such as Weightless, Ingenu, M-Bus, 6Lowpan, but Sigfox and Lorawan are among the most popular at the time being.

\subsection{PWA Underlying Technology in Licenced and License Free Spectrum}

LoRa and Sigfox have a very different approach in terms of technology and business model. Both are opposite technology but give similar outcomes, low power, wide range and cheap design. Sigfox is a French startup company based in Tolouse, founded in 2009. Sigfox acts like a carrier where they sell subscription for sending data via Sigfox proprietary network, thus its network layer specifications is not publicly open [9]. The advantage is that they operate in license free spectrum, using cheap devices and offer an extremely low recurring fee than typical GSM. LoRa is a technology developed by Cycleo in Genoble, France. Semtech acquired the company and created LoRa Alliance ${ }^{\mathrm{TM}}$, an open non-profit association to address the IoT market and drive the success of the LoRa protocol. The alliance collaborates by sharing of knowledge and experience to ensure interoperability among operators in an open universal standard. Various types of organizations and some GSM carriers globally have seen the opportunity to enter the IoT market and subscribed to the alliance. LTE-M is a pure LTE solution optimized for IoT communications and is part of 3GPP Release 12 and 13 that was finalized in 2016. NB-IoT is another 3GPP Release 13 proposal which is not based on LTE. In US mostly, it is not backward compatible with existing LTE, although is integrated in the LTE standard. Both LoRa and Sigfox started their first implementation in Europe. While Sigfox is pioneering the LPWAN technology earlier years back, LoRa had been rolled out in 2016 and currently is in active deployment in few countries globally. For LTE-M, AT\&T and Verizon are working on rolling out the LTE-M initial version in the US, whereas NB-IoT will be Europe focused in which Huawei, Ericsson, Qualcomm, and Vodafone are actively involved in putting the standard together.

\subsection{Sigfox, LoRa, NB-IoT and LTE-M Technical Features}

Sigfox is based on ultra-narrow band (UNB) binary phase-shift keying (BPSK) technology. It is completely asynchronous and transmits at extremely low data rate across over hundreds of uplink channels. Sigfox operates in the $200 \mathrm{kHz}$ of the ISM band; each message is $100 \mathrm{~Hz}$ wide. An uplink message has up to 12 bytes payload with maximum frequency of 140 transmissions per day at a fix bit rate of 100 bps. For a 12 byte data payload, a Sigfox frame will use 26 bytes in total. Sigfox has a very limited downlink channel with 8 bytes payload, available for premium subscribers [11]. It is power efficient as its lightweight protocol to handle very small messages and data to send translated directly into less energy consumption and longer battery life. Sigfox uses star topology network. Each device and base station has a unique Sigfox ID for 
directly to a Sigfox cloud via IP link. The Sigfox station detects, demodulate and report the messages to Sigfox cloud and back end (BE). The BE pushes the messages to business applications or a device transmission authentication. Devices transmit messages to Sigfox station that connected client system. While BE can connect and talk to Sigfox stations, none of them can connect back to the devices.

LoRa is the physical layer of Long Range radio modulation technique integrated with forward error correcting capability. The radio modulation technique is using Chirp Spread Spectrum (CSS) technology that can transmit data with signal strengths below the noise floor. It improves its' link budget and immunity to interference [12], [13]. LoRaWAN is referring to the MAC layer protocol. In LoRa modulation, spreading of the spectrum is achieved by generating a chirp signal that continuously varies in frequency [14]. The communication parameters such as bandwidth (BW), coding rate (CR) and spreading factors (SF) have a significant effect on LoRa deployment scalability. The configuration details are described in paper [15]. By applying different SF, signals are practically orthogonal to each other. This enables different data rates to be demodulated concurrently on the same channel, thus increasing the network capacity. Data rate (DR) and link budget (PRX) can be articulated as in Equation 1 and 2 [16].

$$
\begin{aligned}
& \mathrm{DR}=\mathrm{SF}^{*} \frac{\mathrm{BW}}{2}{ }^{*}{ }^{*} \mathrm{CR} ;\left(\text { Where } \mathrm{CR}=\frac{4}{4+\mathrm{n}}\right) \\
& \mathrm{P}_{\mathrm{RX}}(\mathrm{dBm})=\mathrm{P}_{\mathrm{TX}}(\mathrm{dBm})+\mathrm{G}_{\text {SYSTEN }}(\mathrm{dB})-\mathrm{L}_{\text {SYSTEN }}(\mathrm{dB}) \mathrm{I}_{\text {CHANNEL }}(\mathrm{dB})-\mathrm{M}(\mathrm{dB})
\end{aligned}
$$

LoRaWAN utilizes three device classes to support different types of application scenarios. The different device classes enable trade off to be made between latency and power consumption. Class A consumes the least energy and can be used for applications using sensors or actuators without latency constraint. Downlink is only available shortly after two successful uplink transmissions. In contrast, Class C is the most power starving as it allows end device to listen as often as possible on RX2. This profile matches an application with no latency for downlink communication. Class B will have fixed interval time allocated for the downlink and suitable for scenarios with low latency requirement [18]. LoRa uses pure ALOHA random access scheme for simplicity. However, ALOHA contributes to message lost which leads to capacity drop. LoRa packet structure starts with a preamble field [13], used to synchronize the receiver with the incoming data flow. Next is a header field which provides information about the length and the CR. It uses explicit mode. Third is a payload field, which contains the actual data. If the payload and CR are fix, implicit mode is used by removing the header field, thus reduce the airtime. Cyclic redundancy check (CRC) is the last field, which protects the payload integrity.

LoRaWAN is typically laid out in stars of stars topology [19]. The communication is bi-directional although is uplink dominant. Messages from end devices get encrypted and spread out on different frequency channels and SF to LoRa gateways so each transmission will not interfere with each other. The gateways receive the messages and forward it to a central core network over Ethernet or 3G. The Network Server then routes the messages to the correct end application. There is no association between end devices and gateways, thus any gateways will pick up the messages within its range and forward it to the network server. This feature allows simplicity for nodes mobility as no handover between gateways is required. The network server has the intelligent to perform security check, filter duplicate messages, route the messages to an application server, control radio configuration, send ACKs to gateways and monitor devices and gateways [20]. Security in LoRaWAN is incorporated at the network and application layer to validate the nodes and to protect application data from unauthorized access respectively.

LTE-M is an abbreviated version of LTE-MTC, which allows IoT devices operated on batteries to connect directly to LTE network without a gateway. So to say, LTE-M is a 4G technology downgraded for M2M communications. The LTE channel is made up of $230 \mathrm{kHz}$ spectrum of Physical Resource Block (PRB). LTE-M operates on a 1.4 MHz carrier, thus occupies six PRBs in LTE. For control information, IoT devices will always listen to the six PRB and if to send data will be allocated a number of the PRBs. Power Savings Mode (PSM) and extended discontinuous reception (eDRX) are proposed in Release 12 and 13 respectively to make LTE-M more power efficient [21]. PSM allows the IoT devices to enter a deep sleep mode without having to re-join the network when it wakes up. In eDRX mode, IoT devices only wake up occasionally while connected without losing its network registration [22]. LTE-M introduces half duplex FDD and its band support is limited to sub-GHz band to further reduced cost. LTE-M offers 1Mbps DL and 500 bps UL, which still considered high for M2M applications. LTE-M allows reuse of the LTE installed base and benefits from all the security and privacy of mobile network features, such as entity authentication, confidentiality, data integrity, and mobile equipment identification [23].

A Review of Low Power Wide Area Technology in Licensed and Unlicensed Spectrum ... (Noor Laili Ismail) 
NB-IoT is a new radio access technology which uses an even simpler access scheme, SCFDMA (Single Carrier Frequency Division Multiple Access), thus further reduced cost and device complexity than that of LTE-M, thus optimized it for low end IoT requirements. It is Half Duplex FDD and is based on single PRB operation throughput. As the PHY layer has been changed, fundamental link budget gain is achieved with targeted uplink coverage improvement of $20 \mathrm{~dB}$. The peak bit rate is $500 \mathrm{kps}$ for DL and $40 \mathrm{kps}$ for UL. NB-IoT requires minimum of $180 \mathrm{KHz}$ for both uplink and downlink channels. Therefore, it enables the flexibility of 3 deployments options [20]. GSM operator can choose to replace one GSM carrier of $200 \mathrm{KHz}$ with NB-IoT or an LTE operator can allocate $180 \mathrm{KHz}$ NB-IoT self-contain Physical Resource Block (PRB) inside an LTE carrier for stand-alone and in band deployment respectively. For guard band deployment, NBIoT can use unutilized resource block within LTE guard band frequency with a guaranteed co-existence [24], [25]. Similar to LTE-M, PSM and eDRX Cycle in idle and connected mode are also utilized to give a better power efficiency. eDRX supports device configuration above the previous upper limit of 2.56 seconds [26]. Standard LTE grouped sub-carrier by 12, so individual downlink occupies $15 \mathrm{kHz}$. Sub carrier spacing and slot, sub frame, and frame duration are $0.5 \mathrm{~ms}, 1 \mathrm{~ms}$, and $10 \mathrm{~ms}$ respectively and is based on OFDMA [27]. The uplink is improved from OFDMA to SCFDMA and support both single and multi-tone transmission. Single-tone is based on SCFDMA similar to conventional LTE, with the same $15 \mathrm{kHz}$ subcarrier spacing, 0.5 $\mathrm{ms}$ slot, and $1 \mathrm{~ms}$ sub frame. It achieves the best coexistence performance as it is identical to conventional LTE. Multi-tone transmission supports two options, $15 \mathrm{kHz}$ or optional reduced $3.75 \mathrm{kHz}$ sub-carrier. Equation 3 and 4 below define the uplink (FUL) and downlink (FDL) frequency of NB-IoT [28].

$$
\begin{aligned}
& F_{D L}=F_{D L \_l o w}+0.1\left(\mathrm{~N}_{L}-N_{o f f D L}\right)+0.0025 *\left(2 M_{L}+1\right) \\
& F_{U L}=F_{U L \_l o w}+0.1\left(N_{L L}-N_{o f f U L}\right)+0.0025 *\left(2 M_{L}\right)
\end{aligned}
$$

Where $\mathrm{MDL} / \mathrm{UL}=$ Offset of NB-IoT Channel Number to downlink/uplink, FDL/UL_low= Downlink/uplink operating band, NDL/UL= downlink/uplink E-UTRA Absolute Radio Frequency Channel Number (EARFCN), NoffDL/UL= Minimum range of NDL/UL for downlink/uplink.

To send UL data, User Plane CIoT EPS optimization and the Control Plane CIoT EPS optimization were defined in the evolved packet system (EPS) [28]. UL data is transferred from the eNB (CIoT RAN) to the MME on the Control Plane CIoT EPS optimization. Then, it can either be forwarded to the application server (CIoT Services) through the Service Capability Exposure Function (SCEF) or through Serving Gateway (SGW) and the Packet Data Network Gateway (PGW) path. Similarly, DL transmission takes the same path in reverse direction using signaling radio bearer instead of data radio bearer, thus make it most applicable for transmitting infrequent and small data packets. The SCEF is a new node designed for machine data and only appropriate to deliver non-IP data over control plane. It is responsible for the network services abstract interface such as authentication and authorization, discovery and access network capabilities. User Plane CIoT EPS optimization supports both IP and non-IP data delivery. Data transported on the path to the application server through the SGW and the PGW over radio bearers similar to the conventional data traffic. Although it expedites series of data packets to be delivered, however it also crops some.

\section{RESULTS AND ANALYSIS}

IoT is not a single domination market. Whole range of different used cases and disparity of needs will translate into different architecture and diverse price points. It may create a lot of opportunities to network operators, system integrators or chip companies but it will become a challenge to end users in choosing the right technology that best suit their application scenario [29]. So, it is important to capture the application needs and recognize what each technology platform has to offer in terms of technical features, supporting ecosystem and other deployment factors. Generally, the kind of connectivity required for either smart building, health monitoring or industrial automotive is completely different from each other. In health monitoring that involves life-threatening decisions or critical data streaming applications that cannot tolerate in latency, time and reliability are the key consideration. On the contrary, applications like smart city and environment monitoring that may have thousands of actuators will be focusing on coverage and battery life. A connectivity option that may best suit an application profile may not work properly for the other application scenario. For preliminary review and comparison, information on LoRa, Sigfox, NB-IoT and LTE-M specification are shown in Table 1. 
Table1. Comparison of LoRa, Sigfox, NB-IoT and LTE-M Specifications

\begin{tabular}{|c|c|c|c|c|}
\hline & Sigfox & LoRawan & NB-IoT & LTE-M \\
\hline $\begin{array}{l}\text { Frequency Band } \\
\text { (Country based ISM } \\
\text { Band) }\end{array}$ & $\begin{array}{l}\cdot \mathrm{EU}(868 \mathrm{MHz}) \\
\cdot \mathrm{US}(902 \mathrm{MHz}) \\
\text { [7] }\end{array}$ & $\begin{array}{l}\text { EU }(433,863-870 \mathrm{MHz}) \\
\text { - US (433, 902-928MHz) } \\
\text { - China (470-510MHz, 779- } \\
\text { 787MHz) } \\
\text { - Asean (920-923.5MHz) } \\
\text { [30] }\end{array}$ & $\begin{array}{l}\text { Licensed cellular (LTE) } \\
\text { frequency bands }\end{array}$ & $\begin{array}{l}\text { Licensed cellular } \\
\text { (LTE) frequency } \\
\text { bands }\end{array}$ \\
\hline Bandwidth & $\begin{array}{l}100 \mathrm{~Hz} \\
{[31]}\end{array}$ & $\begin{array}{l}250 \mathrm{kHz} \text { and } 125 \mathrm{kHz} \\
{[31]}\end{array}$ & $\begin{array}{l}200 \mathrm{kHz} \\
{[32]}\end{array}$ & $\begin{array}{l}1.4 \mathrm{MHz} \\
{[32]}\end{array}$ \\
\hline $\begin{array}{l}\text { Uplink \& Downlink / } \\
\text { Duplex Mode }\end{array}$ & $\begin{array}{l}\text { UL: Data } \\
\text { DL: ACK } \\
{[33]}\end{array}$ & $\begin{array}{l}\text { UL: Data } \\
\text { DL: Data + ACK } \\
\text { [33] }\end{array}$ & $\begin{array}{l}\text { Half Duplex } \\
{[26]}\end{array}$ & $\begin{array}{l}\text { Full / Half Duplex } \\
\text { [26] }\end{array}$ \\
\hline Coverage Range & $\begin{array}{l}\text { Urban: } 3-10 \mathrm{~km} \\
\text { Rural : } 30-50 \mathrm{~km} \\
{[14]}\end{array}$ & $\begin{array}{l}\text { Urban: } 3-5 \mathrm{~km} \\
\text { Rural: } 10-15 \mathrm{~km} \\
\text { [9] }\end{array}$ & $\begin{array}{l}15 \mathrm{Km} \\
{[34]}\end{array}$ & $\begin{array}{l}11 \mathrm{Km} \\
{[34]}\end{array}$ \\
\hline Maximum Data Rate & $\begin{array}{l}\text { UL: } 100 \mathrm{bps} \\
\text { DL: } 600 \mathrm{bps} \\
\text { [7] } \\
\text { UL: } 140 \mathrm{msgs}\end{array}$ & $\begin{array}{l}\text { LoRa: } 0.3 \text { - } 37.5 \mathrm{k} \text { bps } \\
\text { FSK: } 50 \mathrm{kbps} \\
\text { [7] }\end{array}$ & $\begin{array}{l}\text { UL: } 250 \mathrm{kbps} \\
\text { DL: } 170 \mathrm{kbps} \\
{[26]}\end{array}$ & $\begin{array}{l}1 \mathrm{Mbps}(\mathrm{FDD}) \\
{[26]}\end{array}$ \\
\hline Max \#msgs/day & $\begin{array}{l}\text { DL: } 4 \text { msgs } \\
{[11][31]}\end{array}$ & Unlimited & Unlimited & Unlimited \\
\hline Battery Life & $\begin{array}{l}90 \text { months } \\
\text { [7] }\end{array}$ & $\begin{array}{l}105 \text { months } \\
{[7]}\end{array}$ & $\begin{array}{l}10 \text { years } \\
{[21]}\end{array}$ & $\begin{array}{l}10 \text { years } \\
{[21]}\end{array}$ \\
\hline $\begin{array}{l}\text { Authentication / } \\
\text { Encryption }\end{array}$ & $\begin{array}{l}\text { No } \\
{[31]}\end{array}$ & $\begin{array}{l}\text { AES - } 128 b \\
{[33]}\end{array}$ & $\begin{array}{l}\text { Yes } \\
{[31]}\end{array}$ & $\begin{array}{l}\text { Yes } \\
{[35]}\end{array}$ \\
\hline Link Budget & $\begin{array}{l}\text { UL: } 158 \mathrm{~dB} \\
{[36]}\end{array}$ & $\begin{array}{l}\text { UL: } 154 \mathrm{~dB} \\
\text { [29] }\end{array}$ & $\begin{array}{l}164 \mathrm{~dB} \\
{[32]}\end{array}$ & $\begin{array}{l}156 \mathrm{~dB} \\
{[32]}\end{array}$ \\
\hline Payload size & $\begin{array}{l}\text { UL: } 12 \text { bytes } \\
\text { DL: } 8 \text { bytes } \\
{[11][33]}\end{array}$ & $\begin{array}{l}\text { 19-250 bytes } \\
{[33]}\end{array}$ & $\begin{array}{l}1600 \text { bytes } \\
{[31]}\end{array}$ & \\
\hline Latency & $\begin{array}{l}10 s \\
{[34]}\end{array}$ & $\begin{array}{l}10 s \\
{[34]}\end{array}$ & $\begin{array}{l}1.4-10 \mathrm{~s} \\
{[31]}\end{array}$ & $\begin{array}{l}10-15 \mathrm{~ms} \\
{[31]}\end{array}$ \\
\hline ADR & No & Yes & No & No \\
\hline Localisation / & No & Yes & No (Release 13) & Full mobility \\
\hline Mobility & [7] & [35] & [26] & {$[26]$} \\
\hline Private Network & No & Yes & No & No \\
\hline Module Cost & $\begin{array}{l}\$ 2-5 \\
{[11]}\end{array}$ & $\begin{array}{l}\$ 2-5 \\
{[11]}\end{array}$ & $\begin{array}{l}\$ 8-12 \\
{[11]}\end{array}$ & $\begin{array}{l}\$ 8-12 \\
{[11]}\end{array}$ \\
\hline
\end{tabular}

Application that transmits infrequently, has rarely burst data with a very limited downlink capability, very small payload size and data rate, Sigfox can be a fantastic choice. Sigfox offers exceptionally low hardware prices, as low as $\$ 2$ per module [11]. In addition to good module price, LoRa also offers good vendors' ecosystem. LoRa is more open as it offered hybrid business models; either deployed your own network and managed them privately or as carriers that are deploying LoRa network. LoRa supports localization and also utilized adaptive data rate to achieve higher data rate. It offers more downlink capability as compared to Sigfox. In many cases, they share a similar application use cases and are excellent option for remote deployment of actuators or sensor based network. The deployments of LoRa and Sigfox are still progressing, and currently the area covers by both are still relatively limited. The shortcoming of operating in unlicensed band spectrum is that it has to deal with certain duty cycle imposed by different regions which restricts the volume and frequency of traffic on devices and gateways, thus drop down the capacity. In some regions, the operating frequency used by both LoRa and Sigfox is also shared by licensed user without duty cycle limit, thus interference can become an issue. LoRa and Sigfox also do not have guaranteed SLA. Future risk of this technology segment is that, as more players of this type of connectivity comes on board, the network could be congested and they may suffer severe interference that place the network performance and reliability at risk. Basic understanding of general IoT segments and application requirements with their use cases are gathered in Table 2, which illustrated the potential best-fit technology platform against the distinct requirements of diverse IoT applications. 
Table 2. General IoT Applications Requirements and Technology Fittings

\begin{tabular}{|c|c|c|c|}
\hline Nature of Applications & Application Domain & Applications Specific & $\begin{array}{l}\text { Best-fit / Potential } \\
\text { Technology }\end{array}$ \\
\hline $\begin{array}{l}\text { - Low periodic data } \\
\text { - Low data rate } \\
\text { - Small payload } \\
\text { - Rarely burst data } \\
\text { - No radical changes of data } \\
\text { - Long battery life } \\
\text { - Does not require QoS } \\
\text { - Limited downlink capability } \\
\text { - Limited LTE coverage } \\
\text { - Low cost }\end{array}$ & $\begin{array}{l}\text { - Agriculture } \\
\text { - Smart City } \\
\text { - Environmental monitoring } \\
\text { - Smart Building } \\
\text { - Manufacturing }\end{array}$ & $\begin{array}{l}\text { - Smart farming } \\
\text { - Smart lighting } \\
\text { - Smart parking } \\
\text { - Smart metering-Temperature \& } \\
\text { humidity monitoring } \\
\text { - Manufacturing Automation }\end{array}$ & Sigfox, LoRa \\
\hline $\begin{array}{l}\text { - Good frequency data } \\
\text { - Good data rate } \\
\text { - Require QoS } \\
\text { - Low latency (sec) } \\
\text { - Dense populated areas with } \\
\text { good LTE coverage }\end{array}$ & $\begin{array}{l}\text { - Environmental monitoring and } \\
\text { control } \\
\text { - Smart City } \\
\text { - Smart Building } \\
\text { - Manufacturing } \\
\text { - Wearable }\end{array}$ & $\begin{array}{l}\text { - Gas/Smoke detectors } \\
\text { - Water level monitoring } \\
\text { - Smart metering } \\
\text { - Street light monitoring and control } \\
\text { - Smart Manufacturing } \\
\text { - Industrial monitoring }\end{array}$ & NB-IoT \\
\hline $\begin{array}{l}\text { - High frequency data } \\
\text { - High data rate } \\
\text { - High QoS } \\
\text { - Very low latency }(\mathrm{ms}) \\
\text { - Dense populated areas with } \\
\text { very good LTE coverage } \\
\text { - Mobility } \\
\text { - Voice data }\end{array}$ & $\begin{array}{l}\text { - Transportation and logistics } \\
\text { - Automotive Telematics } \\
\text { - Wearable } \\
\text { - Real-time grid monitoring } \\
\text { - Security and surveillance }\end{array}$ & $\begin{array}{l}\text { - Asset trackers } \\
\text { - Telematics } \\
\text { - Point of Sale terminals } \\
\text { - Smart watches } \\
\text { - Fitness bands } \\
\text { - Patient monitors } \\
\text { - Alarm panels } \\
\text { - Gas/water meters }\end{array}$ & LTE-M \\
\hline
\end{tabular}

IoT applications profiles which may need higher bandwidth can choose higher bandwidth connectivity solutions such as LTE-M and NB-IoT. NB-IoT is considered to be high end LPWA, targeted to serve ultra-low end IoT application profiles. LTE-M is seems to stand distant apart from the rest of LPWA technology in terms of data rate, potentially at the expense of higher battery consumption and cost. It also supports voice data and is targeted to serve critical or high end IoT applications. Given a country wide deployment in cities area, where power is not really an issue and devices are required to send more frequent data, then NB-IoT offers a superior option at relatively low cost. Applications in this category are such as smart city, smart metering, smart manufacturing and industrial monitoring. LTE-M for sure can serve better for the same application niche but if it doesn't need the kind of bit rate, it may defeat the preliminary purpose of LPWA requirements. So, LTE-M is aimed to serve a critical and higher end applications that require real time communication and far higher bit rate. For instances, applications like gaming, wearable such as patient monitoring, alarm panels and point of sale terminals. Applications that sending streaming data or video such as in security and surveillance, automotive telematic or industrial control can also consider using LTE-M. Unlike LTE-M, NB-IoT does not support devices mobility and localization (as of Release 13). Module cost for NB-IoT and LTE-M are around \$8-\$12 [11], but the current industry target for LPWA to get a reasonable market share is less than $\$ 5$.

The strength of NB-IoT and LTE-M is that they are standardized technology for the industry and can make use of existing infrastructure to be cost effective and faster deployment. They are also supported by a large ecosystem of MNOs which cover nationwide coverage and existing carriers and chip vendors that can provide economy of scale. Cellular technology for quite long has been engaged and gained fair relationship within telecommunication industry players. This advantage will help to provide customers with a high confidence level with respect, reliability and security.Through a high-standardized technology and intervendor interoperability, the technology is capable to guarantee certain level of SLA, which is very important and is absence in the current unlicensed spectrum technologies mentioned above.

\section{CONCLUSION}

Based on the current development of LPWA technology in licensed and license free spectrum, LPWA is seems to gain an increase market share in IoT industry. At present, it is naive to think that an IoT connectivity option can serve every IoT application scenario. Therefore, choosing the best IoT connectivity option for a specific need is very fundamental. This paper compared the differences of Sigfox, LoRa, NB-IoT and LTE-M in terms of their technical features and shortly discussed the pros and cons of their deployment factors. General IoT application requirements are also presented and associated to the deployment factors to give an insight of different applications profiles against the right technology platform, thus provide a simple 
guideline on how to fit the right connectivity features for different applications profiles. It might be a stern competition between the licensed and unlicensed spectrum, but both actually can co-exist as each of them has a different business model and serve a different IoT market segments and application profiles. How all the technologies will co-exist or compete with each other after the arrival of cellular technology are much depend on how they are regulated to fit business requirements and demands, technically and economically.

\section{ACKNOWLEDGEMENT}

The author wish to thank Universiti Teknologi MARA (UiTM) and the Ministry of Higher Education (MoHE) for the support grant of 600-RMI/RAGS 5/3 (35/2015) in this research.

\section{REFERENCES}

[1] Mehaseb, MA, Y Gadallah, H El-Hennawy. WSN Application Traffic Characterization for Integration within the Internet of Things. IEEE 9th International Conference on Mobile Ad-hoc and Sensor Networks. Dalian, China. 2013

[2] Satrya, GB, HT Reda, KJ Woo, PT Daely, SY Shin, S Chae. IoT and Public Weather Data Based Monitoring \& Control Software Development for Variable Color Temperature LED Street Lights. International Journal on Advanced Science, Engineering and Information Technology. 2017; 7(2): 366-372.

[3] Kassim, M, M Ismail, MI Yusof. A New Adaptive Throughput Policy Algorithm On Campus Ip-Based Network Internet Traffic. Journal of Theoretical and Applied Information Technology. 2015; 71(2).

[4] Kassim, M, M Ismail, MI Yusof. Statistical Analysis And Modeling Of Internet Traffic Ipbased Network For TeleTraffic Engineering. ARPN Journal of Engineering and Applied Sciences. 2015; 10(3).

[5] Kassim, M, NA Ayop. Adaptive Policing Algorithms on Inbound Internet Traffic Using Generalized Pareto model. 11th International Conference for Internet Technology and Secured Transactions (ICITST). Barcelona, Spain. 2016.

[6] Boulogeorgos, AAA, PD Diamantoulakis, GK Karagiannidis. Low Power Wide Area Networks (LPWANs) for Internet of Things (IoT) Applications: Research Challenges and Future Trends. ArXiv e-prints, 2016.

[7] Raza, U, P Kulkarni, M Sooriyabandara. Low Power Wide Area Networks: An Overview. IEEE Communications Surveys \& Tutorials, 2017; 19(2): 855-873.

[8] Margelis, G, D Kaleshi, P Thomas. Low Throughput Networks for the IoT: Lessons learned from industrial implementations. IEEE 2nd World Forum on Internet of Things (WF-IoT). 2015.

[9] Centenaro, M, L Vangelista, A Zanella, M Zorzi. Long-range communications in unlicensed bands: The rising stars in the IoT and smart city scenarios. IEEE Wireless Communications, 2016; 23(5): 60-67.

[10] Ali, A, GA Shah, J Arshad. Energy efficient techniques for M2M communication: A survey. Journal of Network and Computer Applications, 2016. 68: 42-55.

[11] Adelantado, F, X Vilajosana, P Tuset-Peiro, B Martinez, J Melia, T Watteyne. Understanding the Limits of LoRaWAN. IEEE Communications Magazine. 2017; 55(9).

[12] Aref, M, A Sikora. Free space range measurements with Semtech Lora technology. 2nd International Symposium on Wireless Systems within the Conferences on Intelligent Data Acquisition and Advanced Computing Systems. Offenburg, Germany. 2014.

[13] Bor, M, J Vidler, U Roedig. LoRa for the Internet of Things. Proceedings of the International Conference on Embedded Wireless Systems and Networks. Graz, Austria. 2016; 361-366.

[14] Augustin, A, J Yi, T Clausen, W Townsley. A Study of LoRa: Long Range \& Low Power Networks for the Internet of Things. Sensors. 2016; 16(9): 466.

[15] Bor, M, U Roedig, T Voigt, J Alonso. Do LoRa Low-Power Wide-Area Networks Scale? The 19th ACM International Conference on Modeling, Analysis and Simulation of Wireless and Mobile Systems. Malta. 2016.

[16] Voigt, T, M Bor, U Roedig, J Alonso. Mitigating Inter-network Interference in LoRa Networks. Computer Science - Networking and Internet Architecture. 2016.

[17] Ducrot, N, D Ray, A Saadani, O Hersent, G Pop, G Remond. LoRa Device Developer Guide. Orange Connected Objects \& Partnerships 2016. Available from: https://partner.orange.com/wp-content/uploads/2016/04/LoRaDevice-Developer-Guide-Orange.pdf.

[18] Neumann, P, J Montavont, T Noël. Indoor deployment of low-power wide area networks (LPWAN): A LoRaWAN case study. IEEE 12th International Conference on Wireless and Mobile Computing, Networking and Communications (WiMob). New York, USA. 2016.

[19] Kim, B, K-i Hwang. Cooperative Downlink Listening for Low-Power Long-Range Wide-Area Network. MDPI Sustainability Journal. 2017; 9: 627.

[20] A technical overview of LoRa ${ }^{\circledR}$ and LoRaWAN ${ }^{\mathrm{TM}}$. Technical Marketing Workgroup 1.0 2015. Available from: https://www.kivi.nl/uploads/media/56992a8894516/LoRaWAN101.pdf.

[21] Zayas, AD, P Merino. The 3GPP NB-IoT system architecture for the Internet of Things. IEEE International Conference on Communications Workshops (ICC Workshops). Paris, France. 2017.

[22] LTE-M - Optimizing LTE for the Internet of Things. Nokia Networks white paper 2015. Available from: https://novotech.com/docs/default-source/default-document-library/lte-m-optimizing-lte-for-the-internet-ofthings.pdf?sfvrsn=0. 
[23] Grant, S. 3GPP Low Power Wide Area Technologies. GSMA Mobile IoT Industry Alignment group 20161 September 2016. Available from: https://www.gsma.com/iot/wp-content/uploads/2016/10/3GPP-Low-PowerWide-Area-Technologies-GSMA-White-Paper.pdf.

[24] Beyene, YD, R Jantti, O Tirkkonen, K Ruttik, S Iraji, A Larmo, T Tirronen, J Torsner. NB-IoT Technology Overview and Experience from Cloud-RAN Implementation. IEEE Wireless Communications. 2017; 24(3): 26-32.

[25] Wang, Y YPE, X Lin, A Adhikary, A Grovlen, Y Sui, Y Blankenship, J Bergman, HS Razaghi. A Primer on 3GPP Narrowband Internet of Things. IEEE Communications Magazine. 2017.

[26] LTE evolution for IoT connectivity. Nokia white paper 2017; Available from: https://resources.ext.nokia.com/asset/200178.

[27] Schlienz, J, D Raddino. Narrowband Internet of Things. Rohde \& Schwarz white paper 2016. Available from: https://www.rohde-schwarz.com/us/applications/narrowband-internet-of-things-white-paper_230854-314242.html.

[28] Sinha, RS, Y Wei, S-H Hwang. A survey on LPWA technology: LoRa and NB-IoT. ICT Express. 2017; 3(1): 1421.

[29] Al-Fuqaha, A, M Guizani, M Mohammadi, M Aledhari, M Ayyash. Internet of Things: A Survey on Enabling Technologies, Protocols, and Applications. IEEE Communications Surveys \& Tutorials. 2015;17(4): 2347-2376.

[30] Lavric, A, V Popa. Internet of Things and LoRa Low-Power Wide-Area Networks: A survey. IEEE International Symposium on Signals, Circuits and Systems (ISSCS). Iasi, Romania. 2017.

[31] Mekki K, Bajic E, Chaxel F, Meyer F. A comparative study of LPWAN technologies for large-scale IoT deployment. ICT Express. 2018.

[32] Lauridsen, M, LC Gimenez, I Rodriguez, TB Sorensen, P Mogensen. From LTE to 5G for Connected Mobility. IEEE Communications Magazine. 2017; 55(3): 156-162.

[33] Nolan, KE, W Guibene, MY Kelly. An evaluation of low power wide area network technologies for the Internet of Things. IEEE 2016 International Wireless Communications and Mobile Computing Conference (IWCMC). Paphos, Cyprus. 2016.

[34] Wang, H, AO Fapojuwo. A Survey of Enabling Technologies of Low Power and Long Range Machine-to-Machine Communications. IEEE Communications Surveys \& Tutorials. 2017; 19(4): 2621-2639.

[35] Silva, JdC, JJPC Rodrigues, AM Alberti, P Solic, ALL Aquino. LoRaWAN - A low power WAN protocol for Internet of Things: A review and opportunities. 2nd International Multi- disciplinary Conference on Computer and Energy Science (SpliTech). Split, Croatia: IEEE. 2017.

[36] Vejlgaard, B, M Lauridsen, H Nguyen, IZ Kovacs, P Mogensen, M Sorensen. Coverage and Capacity Analysis of Sigfox, LoRa, GPRS, and NB-IoT. 2017 IEEE 85th Vehicular Technology Conference (VTC Spring). Sydney, NSW, Australia. 2017. 